\title{
SATELLITE IMPACT PROBABILITIES: ANNUAL SHOWERS AND THE 1965 AND 1966 LEONID STORMS
}

\author{
MARTIN BEECH† \\ Campion College, The University of Regina, Regina, Saskatchewan, Canada \\ RUEDIGER JEHN \\ European Space Operations Centre, Robert-Bosch-Str. 5, 6100, Darmstadt, Germany \\ PETER BROWN and, JAMES JONES \\ Department of Physics, The University of Western Ontario, London, Ontario, Canada, S4S 0A2, \\ N6A 3K7
}

(Received 29 October 1996; revised version received 11 December 1998)

\begin{abstract}
The stream meteoroid impact probability for space platforms is reviewed and found to be very low under normal circumstances. While the literature contains numerous accounts of spacecraft apparently suffering damage and/or interference during meteoroid stream encounters, we find that there is, in fact, very little evidence to support such claims. This conclusion may not be valid, however, during meteor storms, when the flux of visual meteors can increase by factors in excess of $10^{3}$ to $10^{4}$ of that from the sporadic background. Special attention is directed towards the Leonid meteor storms of 1965 and 1966 - the only meteor storms since the dawn of the space age. The space platform impact probabilities during the 1966 storm were small but none negligible, being of order $1 \%$ for an exposed surface area of $2 \mathrm{~m}^{2}$ at a limiting meteoroid mass of $10^{-7} \mathrm{~g}$ (and assuming a stream mass index $s=2.0$ ). The circumstances surrounding the possible encounters of the Pegasus II and III, and Mariner 4 spacecraft with Leonid stream meteoroids are discussed in some detail. While the 1966 Leonid meteor storm is the strongest on record (in the sense of the highest visual meteor rates) no apparent meteoroid inflicted damage to a spacecraft can be unambiguously linked to it. This result is mostly a consequence of the small number and small size of spacecraft in Earth-orbit at the time of the 1966 storm. (C) 1999 Elsevier Science Ltd. All rights reserved
\end{abstract}

\section{MOTIVATION}

From a satellite-operations perspective the space environment is an inherently hostile one from which to work. Not least among the potential threats to the successful completion of any given mission are meteoroid impacts. A spacecraft can suffer impacts from two distinct meteoroid groups: meteoroids from the sporadic background and meteoroids from annual streams. Sporadic meteoroids arrive randomly at the Earth's orbit, throughout the year, and are derived from several broad radiants distributed across the celestial sphere[1]. Stream meteoroids, on the other hand, are highly directional and the Earth only samples significant numbers of such particles for a few days at well-defined times of the year.

Many studies have now shown that under normal conditions, the most likely satellite impactors are sporadic meteoroids, and several detailed models exist to describe the meteoroid flux from this

†Corresponding author. Tel:: + 1306359 1216; fax: + 1 306359 1200; e-mail: martin.beech@uregina.ca. source [2-4]. More recently, however, it has been realized that there are occasions when the flux of stream meteoroids may not be negligible $[5,6]$, and this being particularly so when a stream undergoes outburst, or storm activity. There is no strict definition describing the onset of meteor storm conditions, but we take the attainment of a zenithal hourly rate (ZHR) of 1000 meteors per hour to be the minimum requirement. The ZHR is a derived number constructed to correct the observed hourly rate of meteors to a limiting visual magnitude of +6.5 (at the zenith) assuming that the radiant is directly overhead. The ZHR is a useful number since it allows for the standardization of visual meteor observations, and it can be readily converted to a meteoroid flux if the distribution of meteoroid masses within the stream is known [7].

Meteor storms do not occur very often. During the 19th century six meteor storms were recorded, and so far this century four have been observed. When a meteor storm occurs, the flux of meteoroids capable of generating visible meteors may increase by a factor in excess of $10^{3}$ to $10^{4}$ above that for the sporadic background. Indeed, from our defi- 
Table 1. Summary of Leonid Storms since 1799. The first two columns identify the year and date of the storms. Column three indicates the time at which 55P/Tempel-Tuttle was at perihelion. The time $\Delta t(\mathrm{~h})$ is the time for which shower activity is above ten times the background level, $\mathrm{ZHR}_{\max }$ is the hourly rate at storm maximum and $\delta t$ (days) is the number of days between the passage of the Earth through the descending node of 55P/Tempel-Tuttle, and the time that the comet itself passed through the node [8,9]

\begin{tabular}{|c|c|c|c|c|c|}
\hline Year & Date & Perihelion epoch & $\Delta t(\mathrm{~h})$ & $\mathrm{ZHR}_{\max }$ & $\delta t$ (days) \\
\hline 1799 & Nov. 12.5 & 1800, Mar. 2 & 5 & $>10,000$ & -117 \\
\hline 1832 & Nov. 13.2 & 1833, Jan. 2 & 10 & $>20,000$ & -51 \\
\hline 1833 & Nov. 13.4 & “ & 5 & 100,000 & +308 \\
\hline 1866 & Nov. 13.7 & 1866, Jan. 11 & 5 & 10,000 & +299 \\
\hline 1867 & Nov. 13.8 & " & 5 & $>1,500$ & +664 \\
\hline 1965 & Nov. 17.0 & 1965, Apr. 30 & 17 & $>5,000$ & +196 \\
\hline 1966 & Nov. 17.4 & 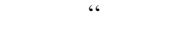 & 5 & 150,000 & +561 \\
\hline
\end{tabular}

nition alone, the meteor rate during a storm must exceed that of the sporadic background by a factor of 100. Under these circumstances the meteoroid impact probability for satellites is dominated, albeit for a short period of time, by stream meteoroids. Of great interest, therefore, is the prediction of times at which meteor storms might occur. Since 1799, eleven storms have been witnessed and of these seven have been due to the Leonid meteoroid stream [6]. At the very least, therefore, we have good reason to investigate the conditions under which Leonid storms occur, and ultimately we need to understand the way in which outbursts and storms might arise from for any given Earth-intersecting meteoroid stream.

Following a brief review, in Section 2, of the most recent Leonid storm-producing epoch, we proceed in Section 3 to review the literature on possible spacecraft interactions. Section 3.1 presents a brief review of annual meteoroid stream characteristics. In Section 3.2 we assess the number and characteristics of spacecraft on orbit between 1964 and 1969, while in Section 3.3 we consider spacecraft impact probabilities with respect to the annual meteoroid streams and the 1965 and 1966 Leonid storms. In Sections 4 and 5 we review in detail two satellite missions that apparently encountered Leonid stream meteoroids. Our conclusions are presented in Section 6.

\section{THE LEONID STORMS-EPOCH 1965}

The parent comet to the Leonid meteoroid stream is $55 \mathrm{P} /$ Tempel-Tuttle. A detailed investigation of the Leonids by Yeomans [8] has revealed that storms are likely to occur whenever the Earth samples meteoroids which are spatially outside of the comet's orbit and temporally behind it. That is, Leonid storms tend to occur when the stream is sampled a year or two after the comet has passed through the nodal point of its orbit. A summary of recent Leonid storm characteristics is given in Table 1 .

The behaviour of annual Leonid shower activity, prior to a definitive epochal storm, is poorly understood at the present time. It appears, however, that the hourly meteor rate at maximum is enhanced each year, for several years either side of the time of Tempel-Tuttle's perihelion passage. Table 2 is a summary of the observational data collected during the 1965 epoch return of Comet Tempel-Tuttle. The observations reveal that enhanced meteor rates were recorded some 4 years before and some 4 years after the time of perihelion passage (April 30, 1965).

It has been found that the activity profile (ZHR vs time) of a meteor storm can be well approximated by a Gaussian function parameterized by the ZHR at the time of maximum and the time during which the activity is ten times the background

Table 2. Leonid meteor shower activity from 1960 to 1970

\begin{tabular}{|c|c|c|c|c|}
\hline Year & Time of nodal crossing & Moon: illuminated fraction & Hourly rate at maximum & Ref. \\
\hline 1960 & Nov. 17.0 & 0.05 & $\sim 10 ?$ & 10 \\
\hline 1961 & Nov. 17.2 & 0.75 & 156 & 11 \\
\hline 1962 & Nov. 17.5 & 0.65 & 20 & 11 \\
\hline 1963 & Nov. 17.8 & 0.02 & $<30$ & 10 \\
\hline 1964 & Nov. 17.0 & 0.95 & $\sim 50$ & 10 \\
\hline 1965 & Nov. 17.3 & 0.40 & $>5000$ & 8 \\
\hline 1966 & Nov. 17.5 & 0.30 & $1.5 \times 10^{5}$ & 10,12 \\
\hline 1967 & Nov. 17.8 & 1.00 & $5-8$ & 12 \\
\hline 1968 & Nov. 17.0 & 0.15 & 80 & 13 \\
\hline 1969 & Nov. 17.3 & 0.60 & $250 \pm 30$ & 14 \\
\hline 1970 & Nov. 17.6 & 0.82 & $\sim 10 ?$ & - \\
\hline
\end{tabular}


level[15]. Estimates of meteoroid fluences during previous Leonid storms are given in Table 3.

\subsection{The 1965 and 1966 Leonid storms}

Leonid meteor storms were clearly and unambiguously observed in 1965 and 1966 . The physical characteristics of these two storms, however, were very different. While the maximum ZHR deduced for the 1965 storm was only $3 \%$ of the maximum ZHR for the 1966 storm, the 1965 storm lasted some 3 times longer than its 1966 counterpart. Radar observations reported by McIntosh and Millman [11] also indicate that the 1966 return was characterized by a relatively high percentage of low mass meteoroids, while that of 1965 was characterized by a higher proportion of greater mass meteoroids. This latter difference is characterized by the stream's mass index, $s$, which is related to the observed population index, $r$, defined as the ratio $r=N\left(M_{\mathrm{v}}+1\right) / N\left(M_{\mathrm{v}}\right)$, where $N\left(M_{\mathrm{v}}\right)$ is the total number of meteors of visual magnitude greater than or equal to $M_{\mathrm{v}}$. The mass index is derived according to the relationship $s=1+2.5 \log _{10}(r)$. The larger the value of $s$, the greater the relative number of lower mass meteoroids.

The cumulative meteoroid flux is related to the mass index through the relation flux $\left(m \geqslant m_{0}\right) \sim$ $m_{0}^{(1-s)}$. Since the cumulative flux for meteoroids brighter than visual magnitude +6.5 , flux $\left(m \geqslant m_{+6.5}\right)$, can be determined from the observations [16], the cumulative flux for meteoroid masses $\geqslant m_{0}$ can be determined according to

$$
\text { flux }\left(m \geqslant m_{0}\right)=\operatorname{flux}\left(m \geqslant m_{+6.5}\right)\left(m_{0} / m_{+0.65}\right)^{1-s}
$$

where $m_{+6.5}$ is the limiting mass for a meteoroid to produce a meteor of visual magnitude +6.5 at the observer's zenith The limiting mass at which a Leonid meteoroid can produce a meteor of visual magnitude +6.5 at the zenith is $10^{-5} \mathrm{~g}$ [17] .

During a typical non-storm return, the Leonid shower is characterized by a mass index of 2 . McIntosh and Millman [11] derived a mass index of 1.6 for the 1965 storm and a mass index of 2.2 for the 1966 storm. McIntosh and Millman [11] also noted that the Leonid outburst of 1961 (see Table 2)

Table 3. Leonid meteoroid fluences. The Gaussian profile parameters $Z H_{\text {max }}$ and $\Delta t$ are taken from Table 2. The limiting meteoroid mass and the meaning of the mass index, $s$, are explained in Section 2.1

\begin{tabular}{ccc}
\hline Year & $\begin{array}{c}\text { Fluence } \\
\left(m \geqslant 10^{-5} \mathrm{~g}\right), s=2.0\end{array}$ & $\begin{array}{c}\text { Fluence } \\
\left(m \geqslant 10^{-5} \mathrm{~g}\right), s=2.5\end{array}$ \\
\hline 1799 & $4.0 \times 10^{-6}$ & $1.6 \times 10^{-5}$ \\
1832 & $1.8 \times 10^{-5}$ & $6.7 \times 10^{-5}$ \\
1833 & $3.8 \times 10^{-5}$ & $1.4 \times 10^{-4}$ \\
1866 & $4.0 \times 10^{-6}$ & $1.6 \times 10^{-5}$ \\
1867 & $7.0 \times 10^{-7}$ & $3.0 \times 10^{-6}$ \\
1965 & $9.0 \times 10^{-6}$ & $3.3 \times 10^{-5}$ \\
1966 & $5.3 \times 10^{-5}$ & $2.0 \times 10^{-4}$ \\
\hline
\end{tabular}

was characterized by a mass index of 1.9 , whereas Millman [18] found that the visual observations collected during the 1969 outburst indicated a high mass index of 2.2. Porubcan and Stohl[19] also found a mass index of $s \sim 2.2-2.4$, from radar observations collected during the 1969 Leonid outburst.

There is a two-fold significance to the observation that the mass index increases during Leonid shower outbursts. Firstly, it means that the true level of visual activity will be slightly more difficult to gauge since local weather effects and/or Moon interference will significantly reduce the observed hourly rates (i.e., the fainter meteors will not be seen). Second, an enhanced mass index implies a greater relative number of low-mass meteoroids and consequently higher satellite impact probabilities.

The difference between the maximum rate and duration of the 1965 and 1966 Leonid storms is easily explained by the fact that the Earth sampled different regions of the stream. The relative differences in the mass index during storms and outbursts is less easily explained, but no doubt relates to the different ejection epochs of the meteoroids, the "sifting" of meteoroid orbits by planetary perturbations and radiation pressure effects.

\section{METEOROID STREAM-SATELLITE INTERACTIONS}

It was clear even before the launch of Sputnik-1 on October 4, 1957 that spacecraft would be subject to impacts from meteoroids [20,21]. This result follows simply from the fact that meteors can be observed in the Earth's atmosphere on any given night of the year. Less simply determined, of course, is an estimate of the cumulative meteoroid flux, for a given limiting mass, in the near-Earth space environment, and indeed this very task constituted the main thrust of the early research programs [22-24].

Early measurements from dust detectors flown on spacecraft suggested that encounters with meteoroid streams were not uncommon. Indeed, Dycus and Bradford [25] argued that Explorer I, launched on February 1, 1958, encountered a previously unknown meteoroid stream during its second and third day in orbit, with a shower radiant near the star $\varepsilon$ Leo. Dycus [26] further noted that the Russian satellite Electron II apparently encountered meteoroids from the same propounded stream on January 31, 1964 and that Explorer XVI also recorded an enhanced number of meteoroid hits between February 5th and 6th in 1963. Dycus and Bradford [25] searched the photographic meteor orbit data base of McCrosky and Posen [27] and found eleven photographic meteors with orbital characteristics consistent with a radiant near $\varepsilon$ Leo. To date no visually obtained data have been presented to support the conjecture of a meteor shower, with a radiant near $\varepsilon$ Leo, in early 
February - it should be noted, however, that many minor meteor showers, with radiants near the ecliptic, are active at the time of interest [28]. While the lack of supportive visual observations is not fatal to the idea that Explorer I, Electron II and Explorer XVI encountered a meteoroid stream, caution must be extended towards the result since the dust-detectors on the first two craft were acoustic ones. This is a problem since Nilsson [29] has shown that such detectors were susceptible to poor calibration and high levels of thermally induced noise. The fact that three independent spacecraft recorded high impact rates from the same general direction in three different years is, however, intriguing.

No clear-cut evidence has ever been presented to show that a given spacecraft has suffered critical structural damage from a meteoroid impact. There continue to be a number of cases, however, where meteoroid impacts are undeniably implicated as damaging agents (see below). One of the earliest claims of meteoroid impact induced damage was that described by Kellogg[30] who attributed anomalies experienced by Explorer III in 1959 to the passage of the craft through the $\eta$-Aquarid stream. Indeed, it was suggested that two wire detectors were severed during the encounter. Certainly, the $\eta$-Aquarid meteor shower is one of the more prominent annual meteor showers, but with a flux of some $5 \times 10^{-12}$ meteoroids $/ \mathrm{m}^{2} / \mathrm{s}$ at shower maximum, it is difficult to see how any spacecraft damage, let alone two wire severs, could result from a stream encounter. Wire sever probabilities are further discussed in [31].

Of interest to the main aims of this study Alexander et al. [32] have reported that the Vanguard III satellite, launched on September 18, 1959 , detected a significant number of meteoroid hits from the Leonid stream between November 16 and 18, 1959. The authors state that the Vanguard III dust-detector had a limiting mass sensitivity of $\sim 10^{-9} \mathrm{~g}$, and that the average flux recorded during a 70-h interval commencing November 16, 1959 was 0.2 meteoroids $/ \mathrm{m}^{2} / \mathrm{s}$.

The proposed encounter between the Leonid stream and Vanguard III is both interesting and tantalizing. The key question, of course, is can we believe it. There are several critical reasons why, given our advantage of hindsight, we should not believe the analysis of Alexander et al. [32]. Firstly, caution must be directed towards the observations because the Vanguard III detector was an acoustic one and hence susceptible to unqualified noise from thermal variations. Second, on dynamical grounds we expect that solar radiation pressure will rapidly scour the Leonid stream of all meteoroids with masses less than $\sim 10^{-8} \mathrm{~g}$ [33]. Thirdly, and most critically, if we assume that the cumulative flux varies as a power law with meteoroid mass as $m^{1-s}$, where $s$ is the stream's mass index, then the implied flux for meteoroids of mass $m \geqslant 10^{-5} \mathrm{~g}$ with $s=2.0$, is $\sim 2 \times 10^{-5}$ meteoroids $/ \mathrm{m}^{2} / \mathrm{s}$. This flux in turn implies a visual meteor rate of some 100 million per hour! Needless to say, no such meteor rates were observed in 1959 and clearly we cannot accept the Vanguard III results at face value. The apparent Leonid stream encounter was, we suggest, the result of an overly optimistic interpretation of data derived from a noisy detector.

Sounding rockets have also been used to derive estimates of meteoroid fluxes in the Earth's upper atmosphere. Berg and Meredith [34], for example, reported a large number of impacts during an Aerobe NIL-25 flight on November 17, 1955 (a point not missed by Alexander et al. [32]). But again, no enhanced activity was recorded for the Leonid stream in that year and we are obliged to dismiss the result as spurious. Venus Flytrap, dustcapture experiments were carried by sounding rockets to altitudes of about $100 \mathrm{~km}$ shortly after the times of peak activity during the 1965 and 1966 Leonid displays [35]. While the Leonid rates were most definitely at storm levels in 1965 and 1966 no Leonid meteoroids were captured during any of the flights. These null-capture results may, however, be mostly related to the very small capture area, $0.04 \mathrm{~m}^{2}$, of the experiments.

\subsection{Meteoroid stream fluxes}

It is now a well-established observation that the annual meteoroid streams do not typically contribute in any significant manner to the meteoroid flux experienced by Earth-orbiting spacecraft [36,3]. The cumulative flux of Leonid meteoroids, for example, with masses $\geqslant 10^{-5} \mathrm{~g}$, at the time of maximum activity, during non-storm returns is $\sim 10^{-12}$ meteoroids $/ \mathrm{m}^{2} / \mathrm{s}$. The cumulative flux of Leonid meteoroids during non-storm years is, therefore, some four orders of magnitude smaller than that from sporadic meteoroids with masses $\geqslant 10^{-5} \mathrm{~g}[3]$. While the flux of sporadic meteoroids may dominate that of the annual streams, this is not to say that spacecraft cannot be hit and damaged by stream meteoroids. Indeed, there exist good reason to believe that the mission-ending anomaly experienced by the OLYMPUS communications satellite was initiated by a meteoroid impact. The craft was possibly struck by a Perseid meteoroid on August 12, 1993 [37]. Even so, belief in the Perseid meteoroid impact hypothesis is only sustainable because the observed Perseid meteor rates were some two times higher than normal at the time of the anomaly $[38,39]$.

Figure 1 shows the annual variation of the cumulative flux of stream meteoroids, at shower maximum, to a limiting mass of $10^{-5} \mathrm{~g}$. The streams are modeled according to the procedure and data of [14]. It is clear from the Figure that the cumulative flux of sporadic meteoroids, to a limiting mass of $10^{-5} \mathrm{~g}$, dominates that of the streams at all times. Indeed, we note that the only reason annual 


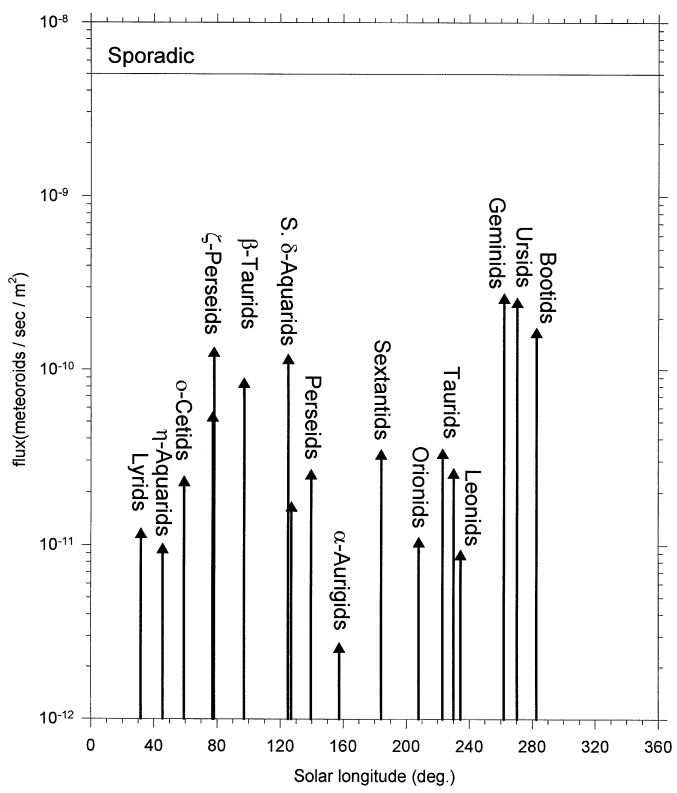

Fig. 1. Cumulative fluxes at maximum for the main annual meteor showers. The time axis is in solar longitude and the limiting mass is $10^{-5} \mathrm{~g}$. The flux is calculated according to the data in [14] and the sporadic flux is taken from [3].

meteor showers are so "obvious" to visual observes is because their constituent meteors appear to radiate from the same region of the sky (the radiant) and because stream meteoroids tend to have high Earth-encounter velocities. The latter point is particularly important since the luminous efficiency is proportional to the fourth power of the velocity [40]. The cumulative flux at shower maximum, to a limiting mass of $10^{-5} \mathrm{~g}$, for the major annual meteor showers is given in Table 4 .

The interesting point illustrated by the entries in Table 4 is that the meteoroid flux at maximum and the annual fluences of what are comparatively minor visual showers can, on occasion, match those of the most active visual showers. The fluence of Ursid meteoroids, for example, is greater than that of the Perseids even though the Perseid shower lasts about five times longer and produces nearly ten times more visual meteors at shower maximum. The reason for this result is that the Ursid stream has a much higher mass index than the Perseid stream, and because the Ursids have a low Earth encounter velocity.

\subsection{Satellites on orbit $1964-1969$}

The number of objects on orbit during the Leonid returns from 1964 to 1968 has been derived from DISCOS (Database and Information System Characterizing Objects in Space) [41]. The database has been searched to determine both the number and surface area of objects in low Earth-orbit (LEO) in the time interval of interest. An orbital altitude of $2000 \mathrm{~km}$ has been set as the LEO limit - all other objects simply being classified as outside of LEO. Table 5 is a summary of the data-

Table 4. Cumulative flux at maximum of the major annual meteoroid streams. All of the fluxes have been reduced to the same limiting mass of $10^{-5} \mathrm{~g}$ assuming a constant mass index. The sporadic meteoroid flux at the chosen limiting mass is $5.0 \times 10^{-9} \mathrm{meteoroids} / \mathrm{m}^{2} / \mathrm{s}$

\begin{tabular}{lcccc}
\hline Stream & Mass index & Flux $\left(m \geqslant 10^{-5}\right)\left(\right.$ meteoroids $\left./ \mathrm{m}^{2} / \mathrm{s}\right)$ & Duration (days) & Fluence $\left(m \geqslant 10^{-5}\right)\left(\mathrm{meteoroids} / \mathrm{m}^{2}\right)$ \\
\hline Bootids & 2.0 & $1.4 \times 10^{-10}$ & 2.6 & $1.5 \times 10^{-9}$ \\
Lyrids & 2.1 & $1.2 \times 10^{-11}$ & 13.0 & $1.1 \times 10^{-9}$ \\
$\eta$ Aquarids & 2.1 & $9.2 \times 10^{-12}$ & 47.3 & $2.4 \times 10^{-9}$ \\
Arietids & 2.1 & $1.4 \times 10^{-10}$ & 41.0 & $3.1 \times 10^{-8}$ \\
$\delta$ Aquarids S. & 1.3 & $6.2 \times 10^{-12}$ & 30.2 & $1.4 \times 10^{-9}$ \\
Perseids & 2.0 & $2.1 \times 10^{-11}$ & 22.5 & $2.2 \times 10^{-9}$ \\
Orionids & 2.2 & $9.8 \times 10^{-12}$ & 28.6 & $1.7 \times 10^{-9}$ \\
Leonids & 2.0 & $3.4 \times 10^{-12}$ & 8.5 & $1.8 \times 10^{-10}$ \\
Geminids & 2.0 & $2.0 \times 10^{-10}$ & 8.3 & $7.5 \times 10^{-9}$ \\
Ursids & 2.3 & $1.7 \times 10^{-10}$ & 4.5 & $6.0 \times 10^{-9}$ \\
\hline
\end{tabular}


Table 5. Number and total cross-section area of objects in Earth orbit between November 1964 and November 1968. The West Ford Needles have not been included in the sum of cross-section areas

\begin{tabular}{|c|c|c|c|c|c|}
\hline & 1964 & 1965 & 1966 & 1967 & 1968 \\
\hline Number of objects in LEO & 556 & 1381 & 1492 & 1531 & 1746 \\
\hline Number of objects outside LEO & 104 & 144 & 194 & 262 & 327 \\
\hline Total number of objects & 660 & 1525 & 1686 & 1793 & 2073 \\
\hline West Ford Needles & 150 & 151 & 151 & 152 & 151 \\
\hline Cross-section area in LEO $\left(\mathrm{m}^{2}\right)$ & 2664 & 3516 & 3785 & 3921 & 3555 \\
\hline Cross-section area outside LEO $\left(\mathrm{m}^{2}\right)$ & 172 & 247 & 1061 & 1195 & 1290 \\
\hline Total cross-section area $\left(\mathrm{m}^{2}\right)$ & 2836 & 3763 & 4846 & 5116 & 4845 \\
\hline
\end{tabular}

base search. We have not included the West Ford Needles [42,43] in either the number count or the cross-section area sum. The West Ford Needles were initially composed of some 350 million, $2 \mathrm{~cm}$ long wire dipoles which circled the Earth at an altitude of $3900 \mathrm{~km}[44,45]$ Likewise we have not included the Pageos fragments in the number count. These fragments have a launch date of June 1966, but were not created until July 1975.

The cross-section area of each fragment in Earthorbit between November 1964 and November 1968 is either based upon the observed radar cross-section, when known, or is assumed to be that of a sphere with a diameter equal to the average spacecraft dimensions. The total surface area of spacecraft in Earth-orbit will be of approximately 4 times the cross-section areas given in Table 5. It is interesting to observe that the surface area of objects in LEO was dominated from 1964 to 1968 by just two spacecraft-Echo I and Echo II. In 1964 these two craft accounted for $76 \%$ of the total cross-section area in LEO, while in 1967 they accounted for over $50 \%$ of the total. The large decrease in cross-sectional area between 1967 and 1968 is mostly due to the re-entry of Echo 1 in May 1968.

A total of 16 on-orbit fragmentation events were recorded between November 5, 1964 and October 4, 1969. None of these events, however, occurred near the maximum of a Leonid shower or storm, and consequently we cannot directly attribute any of the breakups to Leonid meteoroid impacts.

\subsection{Impact probabilities}

It is a non-trivial exercise to calculate the meteoroid impact probabilities for specific spacecraft in
Earth orbit. Here we make a number of simplifying assumptions and present representative impact probabilities rather than discuss specific spacecraft scenarios. We assume, for example, that a spacecraft presents a constant surface area to the meteoroid stream radiant, and we do not account for Earth shielding and/or gravitational focusing. For a given meteoroid stream fluence, $F_{\mathrm{S}}$, and exposed surface area, $A$, the number of expected impacts $N$ will be the product $F_{\mathrm{S}} A$. This can be converted to a percentage probability by multiplying $N$ by 100 . In general, the probability that there will be one or more meteoroid impacts during a particular stream crossing is

$$
\operatorname{Ip}(\%)=100 \times \text { fluence } \times \text { Area }
$$

Since a typical spacecraft will not present a constant surface area towards a stream radiant and because of the intermittent effects of Earth shielding, the impact probability $\mathrm{Ip}(\%)$ will be an upper bound estimate for the given fluence.

The probability of exactly $\mathrm{m}$ meteoroid impacts during the passage of the Earth through a given meteoroid stream can be calculated from Poisson statistics as

$$
P(m)=\left(N^{\mathrm{m}} / m !\right) \mathrm{e}^{-\mathrm{N}}
$$

In the case of the major annual meteoroid streams the fluences are given in Table 4, and for the Leonid storms the fluences are given in Table 3. The area can be either that of a specified spacecraft or the total spacecraft surface area given in Table 5 . The impact probabilities arising during the annual meteoroid streams is given in Table 6 , while the

Table 6. Spacecraft impact probabilities during annual meteor showers. The meteoroid impact velocities and the ZHR at maximum are taken from [14]. The impact probability is that for an exposed surface area of $1 \mathrm{~m}^{2}$ and the stream fluences are taken from Table 4 . The limiting mass for the calculations is $10^{-5} \mathrm{~g}$

\begin{tabular}{lccc}
\hline Stream & Velocity $(\mathrm{km} / \mathrm{s})$ & ZHR at maximum & Impact probability $(\%)$ \\
\hline Bootids & 43 & 133 & $1.5 \times 10^{-7}$ \\
Lyrids & 49 & 13 & $1.1 \times 10^{-7}$ \\
$\eta$ Aquarids & 66 & 37 & $2.4 \times 10^{-7}$ \\
Arietids & 38 & 54 & $3.1 \times 10^{-6}$ \\
$\delta$ Aquarids S. & 43 & 11 & $1.4 \times 10^{-7}$ \\
Perseids & 61 & 84 & $2.2 \times 10^{-7}$ \\
Orionids & 67 & 25 & $1.7 \times 10^{-7}$ \\
Leonids & 71 & 23 & $1.8 \times 10^{-8}$ \\
Geminids & 36 & 88 & $7.5 \times 10^{-7}$ \\
Ursids & 35 & 12 & $2.3 \times 10^{-7}$ \\
\hline
\end{tabular}


impact probabilities resulting from the 1965 and 1966 Leonid storms are given in Table 7.

Since the impact probability scales linearly with exposed surface area (see eqn (2) we can see from Table 6 that for an exposed cross-section area of say $100 \mathrm{~m}^{2}$, the impact probability for the annual streams never rises above $10^{-4}$ per cent per year. At this level it is highly unlikely that a spacecraft will suffer a single stream meteoroid hit during its entire mission lifetime.

The impact probabilities under storm conditions (Table 7) are, as one would expect, higher than those from the annual streams. Even so, the impact probability, to a limiting mass of $10^{-5} \mathrm{~g}$, on a small sized (area $\sim 10 \mathrm{~m}^{2}$ ) spacecraft is quite low, and this being so even under the extreme 1966 Leonid storm conditions. Large spacecraft, such as Echo I and Echo II, clearly fare less well under storm conditions and impact probabilities of several tens of per cent can be realized.

Impact probabilities to lower limiting meteoroid masses can be calculated with the aid of eqn (1). At a limiting mass of $10^{-7} \mathrm{~g}$, for example, the impact probabilities increase by a factor of 100 for $s=2.0$ and by a factor of 1000 for $s=2.5$. Clearly, since the number of meteoroids increases with decreasing limiting mass (that is, to a limiting mass set by radiation pressure), the impact probabilities will correspondingly rise. At a limiting mass of $10^{-7} \mathrm{~g}$ we see that the impact probability for a spacecraft with a cross-sectional area of $10 \mathrm{~m}^{2}$ was $\sim 1 \%$ during the 1965 Leonid storm $(s=2.0)$ and $\sim 200 \%$ during the 1966 Leonid storm $(s=2.5)$. The impact probabilities during the 1964, 1967 and 1968 Leonid returns will be a factor of $10^{3}$ to $10^{4}$ times smaller than those given in Table 7 .

Four commercial communication satellites had been successfully placed in geostationary Earth orbit by the close of 1964 . These were the three Syncom satellites and Intelsat-1 (Early Bird). Syncom 1 failed shortly after launch, but the remaining craft were fully functional during the 1965 and 1966 Leonid storms [46]. The impact probabilities for the GEO craft are given in Table 8. At a limiting mass of $10^{-7} \mathrm{~g}$ and an assumed mass index $s=2$, the impact probabilities are of order 0.2 to $1.2 \%$. At these impact probability levels we would not expect any spacecraft damage, and
Table 8. Communication satellites in geostationary orbit during the 1965 and 1966 Leonid meteor storms. The impact probabilities are calculated according to the $s=2.0$ fluences given in Table 3 and adjusted to a limiting mass of $10^{-7} \mathrm{~g}$

\begin{tabular}{lccc}
\hline Spacecraft & Area $\left(\mathrm{m}^{2}\right)$ & $\mathrm{Ip}(\%) 1965$ & $\mathrm{Ip}(\%) 1966$ \\
\hline Syncom 1 & 2.22 & 0.20 & 1.18 \\
Syncom 2 & 2.22 & 0.20 & 1.18 \\
Syncom 3 & 2.22 & 0.20 & 1.18 \\
Early Bird & 2.15 & 0.19 & 1.14 \\
\hline
\end{tabular}

indeed, none was reported. It is worth noting, however, that if we assume a mass index of $s=2.5$ for the 1966 Leonid storm, then the impact probability for a Syncom satellite is of order $44 \%$. At this level of probability, we might well have expected at least one hit on one of the GEO satellites.

We can also look at the inverse problem and ask what is the visual ZHR that produces an impact probability of $99 \%$, at a limiting mass of $10^{-7} \mathrm{~g}$, for a Syncom satellite. With a mass index $s=2$ and a storm duration of $5 \mathrm{~h}$ we find that a ZHR at maximum of order $10^{7}$ meteors per hour is required. This required ZHR is some 70 times higher than the peak rate observed in 1966 .

In summary, our assessment of impact probabilities for Leonid meteoroids during the period 1964 to 1968 indicates that the only year in which impacts were probable was 1966 . The Leonid storm of 1966 holds the record for being the strongest (in the sense of greatest ZHR) meteor storm ever recorded. Our impact probability analysis suggests that in 1966 many Leonid meteoroid impacts may have occurred, at a limiting mass of $10^{-7} \mathrm{~g}$, and yet the data also indicate that no events leading to mission termination, or fragmentation were recorded. This is an encouraging result from a satellite operations point-of-view, and it suggests that future Leonid storms might well be 'weathered' by adopting area minimization maneuvers (e.g., feathering solar arrays towards the stream radiant). We have also shown in this section that the impact probabilities for annual stream meteoroids, over a satellite's mission lifetime, are very small to negligible.

\section{THE PEGASUS PROGRAM}

In total three Pegasus satellites were deployed in Earth-orbit. The craft were essentially modified

\begin{tabular}{|c|c|c|c|c|}
\hline \multirow[t]{2}{*}{ Cross-section area $\left(\mathrm{m}^{2}\right)$} & \multicolumn{2}{|c|}{1965} & \multicolumn{2}{|c|}{1966} \\
\hline & $s=2.0$ & $s=2.5$ & $s=2.0$ & $s=2.5$ \\
\hline 10 & 0.01 & 0.03 & 0.05 & 0.20 \\
\hline Echo I (707) & 0.64 & 2.33 & 3.75 & 14.14 \\
\hline Echo II (1320) & 1.19 & 4.36 & 7.00 & 26.40 \\
\hline Objects in LEO & 1.34 & 4.91 & 9.32 & 35.16 \\
\hline Objects outside LEO & 0.22 & 0.82 & 5.62 & 21.22 \\
\hline
\end{tabular}


Table 9. Summary of Pegasus II and III data for the 1965 Leonid return. The detector limiting masses are taken from [49]. $N$ is the number of impact events recorded in the 24-h period starting midnight 17 November 1965

\begin{tabular}{lccccc}
\hline Spacecraft & Detector $(\mu \mathrm{m})$ & Area $\left(\mathrm{m}^{2}\right)$ & Mass Limit $(\mathrm{g})$ & Events & Fluence $\left(\mathrm{met} / \mathrm{m}^{2}\right)$ \\
\hline Peg II + III & 410 & 342 & $5 \times 10^{-8}$ & 4 & 0.012 \\
Peg II & 38 & 7.5 & $5 \times 10^{-11}$ & 4 & 0.533 \\
Peg III & 38 & 7.5 & $5 \times 10^{-11}$ & 2 & 0.267 \\
\hline
\end{tabular}

Saturn-1 rocket bodies equipped with large extendible wings and the program was specifically designed to study meteoroid fluxes in LEO. The three craft were launched on February 16, May 25, and July 30, 1965. Data was gathered from the satellites through to the end of December 1965 [47,48]. The primary aim of the Pegasus program was to determine the meteoroid penetration frequencies for three different thicknesses of aluminum foil. The Pegasus data is of particular interest to this study since both Pegasus II and III were on-orbit and functional during the 1965 Leonid storm.

Clifton and Nauman [48] found that during the 24-h period beginning midnight on November 17 , 1965 a total of four events were recorded by the $410 \mu \mathrm{m}$ detectors aboard Pegasus II and III. The $38 \mu \mathrm{m}$ detector abroad Pegasus II recorded four events in the same time interval, while that on Pegasus III recorded two events. The latter two rates are typical of that expected for the detectors on any given day, while that for the $410 \mu \mathrm{m}$ detector exceeds the expected daily rate by more than $2 \sigma$.

Since we know the detector areas and the number of recorded meteoroid hits, we can derive an estimate of the apparent meteoroid fluence. Table 9 is a summary of the November 17, 1965 results for Pegasus II and III.

In order that the fluence estimates be of some practical use, we need to determine the penetration mass threshold for the detectors. A calibration of the Pegasus foils has been published by Fechtig et al. [49] and from their data we estimate that at 20 $\mathrm{km} / \mathrm{s}$ the sensitivity threshold is $10^{-6} \mathrm{~g}$ for the $410 \mu \mathrm{m}$ foil and $10^{-9} \mathrm{~g}$ for the $38 \mu \mathrm{m}$ foil. At 70 $\mathrm{km} / \mathrm{s}$, the encounter velocity appropriate to Leonid meteoroids, the threshold masses are a factor of 20 smaller than at $20 \mathrm{~km} / \mathrm{s}$. Figure 2 shows the three Pegasus data points, from Table 9, in the cumulative flux vs mass diagram. It was noted in Section 3 that the lower mass limit to meteoroids in the Leonid stream is $\sim 10^{-8} \mathrm{~g}$, and we also see from Fig. 2 that the $38 \mu \mathrm{m}$ data points are in good agreement with the Cour-Palais model. We assume, therefore, that the $38 \mu \mathrm{m}$ foil penetrations were due to sporadic meteoroid hits. The data point for the $410 \mu \mathrm{m}$ detector, however, is a little more intriguing. Certainly, it is possible that the apparently enhanced number of hits was due to detector noise, and it is not impossible that all of the hits were sporadic meteoroids. The impact probability implied by the visual observations for the Pegasus II and III detector area, is about $70 \%$ at a limiting meteoroid mass of $5 \times 10^{-8} \mathrm{~g}(s=2.0)$ and consequently one would not expect to see evidence for more than one Leonid meteoroid hit during the storm. The data is certainly not conclusive, but it is consistent with the argument that at least one of the recorded hits on the $410 \mu \mathrm{m}$ detectors was due to a Leonid meteoroid.

\section{MARINER IV STREAM ENCOUNTERS}

Mariner 4 was launched on November 28, 1964 and was the first spacecraft to return television pictures of the surface of Mars to Earth [50]. The spacecraft encountered Mars in July of 1965, and

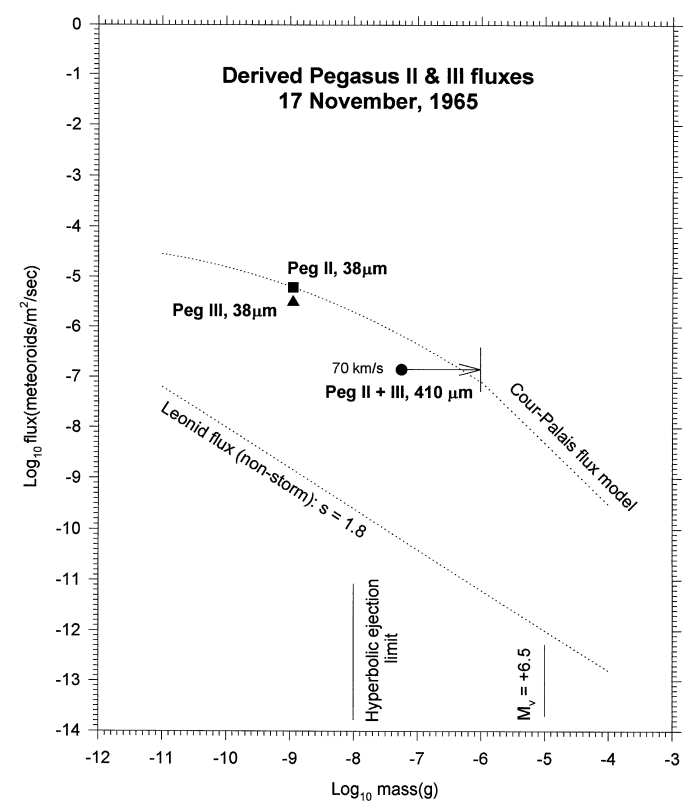

Fig. 2. Cumulative meteoroid flux as derived from Pegasus II and III during the 24 -h period commencing November 17, 1965. The Cour-Palais [2] cumulative flux model for sporadic meteoroids, and the cumulative flux for nonstorm Leonid meteoroids at the time of shower maximum are also shown in the diagram. The hyperbolic ejection limit at $10^{-8} \mathrm{~g}$ corresponds to the minimum mass that a Leonid meteoroid can have and still remain in the stream. The $M_{\mathrm{v}}=+6.5$ limit indicates the threshold at which Leonid meteoroids can produce a visible meteor in the Earth's atmosphere, when seen at an observer's zenith. See text for details. 
Table 10. Post-Mars encounter parameters for Mariner 4

\begin{tabular}{ll}
\hline Semi-major axis (AU) & 1.340843 \\
Eccentricity & 0.17322007 \\
Inclination (deg.) & 2.5437401 \\
Longitude of ascending node (deg.) & 226.75545 \\
Argument of perihelion (deg.) & 200.64908 \\
Time of perihelion passage & $07: 25: 19,16$ November, 1964 \\
\hline
\end{tabular}

Table 11. The position of Mariner 4 at the times of purported stream encounters

\begin{tabular}{lccc}
\hline Date & $\begin{array}{c}\text { Heliocentric } \\
\text { distance (AU) }\end{array}$ & $\begin{array}{c}\text { Ecliptic } \\
\text { longitude (deg.) }\end{array}$ & $\begin{array}{c}\text { Ecliptic } \\
\text { latitude (deg.) }\end{array}$ \\
\hline 1967, Sept 15. & 1.27 & 344.58 & $2.25 \mathrm{~N}$ \\
1967, Dec. 10. & 1.11 & 53.72 & $0.31 \mathrm{~S}$ \\
\hline
\end{tabular}

thereafter adopted an orbit with a perihelion distance of 1.108 AU. The parameters of the "postencounter" orbit are given in Table 10 and are taken from [51].

One of the experiments carried aboard the Mariner 4 craft was a cosmic-dust detector [52]. The detector was a combined acoustical plate and capacitor film sensor. Since the minimum mass detection limit of the detector was not known, the system simply counted meteoroid hits above a limiting momentum threshold determined by the acoustical transducer. Following the Mars encounter of July 1965, re-acquisition of Mariner 4 telemetry began in March 1966, but data recovery was intermittent until early 1967[53]. From mid-July to midOctober, 1967 telemetry coverage was fairly complete. All spacecraft operations were suspended, however, on December 20, 1967.

According to [53] and as documented in Aviation Week and Space Technology [54] Mariner 4 encountered meteoroid streams on September 15, 1967 and December 10, 1967. The reasons for believing that meteoroid streams were encountered on these dates were, (a) enhanced numbers of apparent meteoroid hits, and (b) the detection of perturbative torque's about the spacecraft's roll axis. The position of Mariner 4, in ecliptic coordinates, can be calculated from the data in Table 10 and are given in Table 11 .

The idea that Mariner 4 encountered two meteoroid streams in rapid succession is not, from our present perspective, easy to believe. Given that no detailed analysis of the propounded Mariner 4 stream encounters has ever been published we present a study of these events below.

\subsection{Re-analysis of the Mariner 4 anomalies}

The exact positions of Mariner 4 at the times of the propounded encounters are well known and are given in Table 11. The distances of closest approach between Mariner 4 and all of the major annual meteoroid streams [28] have been calculated. Figures 3 and 4 through 5 show the conditions of closest encounter (to a limiting distance of $0.6 \mathrm{AU}$ )

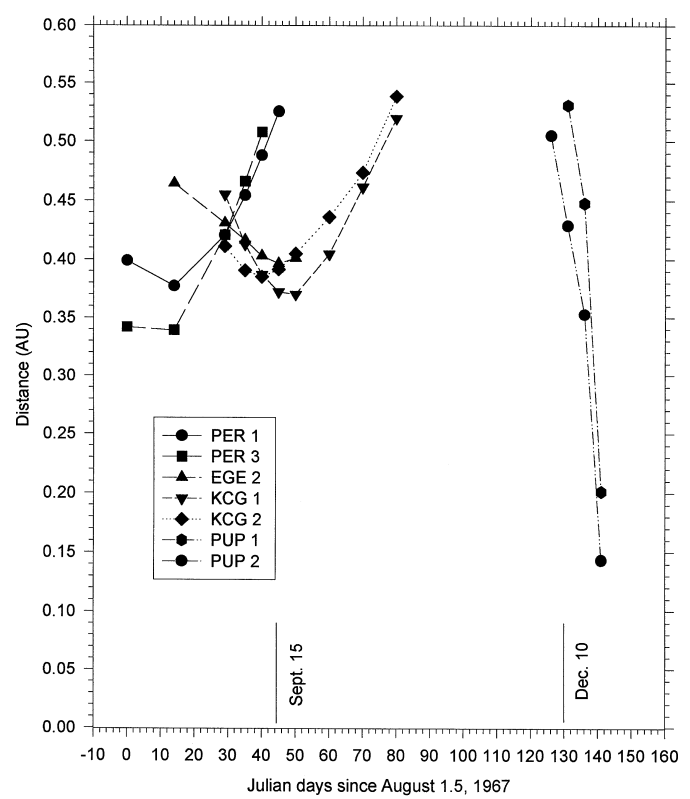

Fig. 3. Meteoroid stream encounter conditions for Mariner 4 between August 1.5 and December 30.5, 1967. See Table 12 for stream identification details. Time is measured in days and the distance of Mariner 4 to the mean stream orbit is given in astronomical units.

for all of the annual meteoroid streams between August 1 and December 31, 1967. The orbital characteristics of the meteoroid streams are taken from Rendtel et al. [28] and are reproduced here in Table 12. We find that Mariner 4 was positioned within $0.5 \mathrm{AU}$ of three meteoroid streams; the Perseids, the epsilon-Geminids and the kappa-

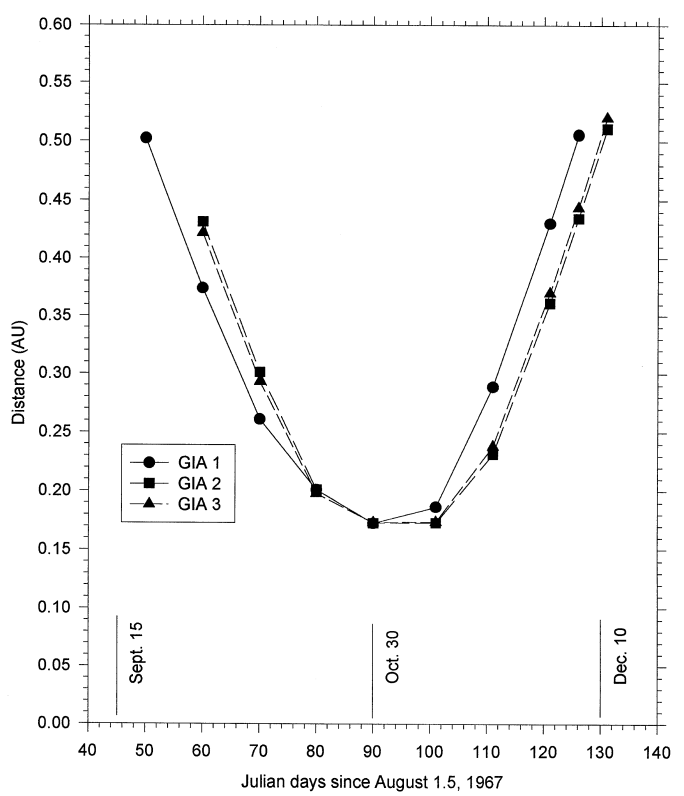

Fig. 4. Same as Fig. 3, but showing the encounter conditions for the Giacobinid meteoroid stream. 
Table 12. Mean meteoroid stream characteristics. The orbital parameters are taken from Rendtel et al. [28], and the reader is directed to this reference for further details. The orbit for 55P/Tempel-Tuttle is taken from Yeomans [8]. The column labeled "Method" refers to the observational technique used in the survey

\begin{tabular}{|c|c|c|c|c|c|c|c|}
\hline Stream & ID & $\Omega$ & $\omega$ & $i$ & $\mathrm{e}$ & $a$ & Method \\
\hline Perseids & PER 1 & 139.6 & 150.5 & 113.2 & 0.996 & 81 & photographic \\
\hline “ & PER 3 & 136 & 151 & 113 & 0.91 & 11 & radar \\
\hline$\varepsilon$-Geminids & EGE 2 & 203 & 223 & 175 & 0.75 & 3.6 & " \\
\hline$\kappa$-Cygnids & $\mathrm{KCG} 1$ & 147.7 & 199.8 & 38.2 & 0.769 & 4.50 & photographic \\
\hline " & KCG 2 & 142.0 & 197.7 & 33.0 & 0.718 & 3.51 & $" “$ \\
\hline Puppid-Velids & PUP 1 & 82 & 0 & 70 & 0.2 & 1.3 & radar \\
\hline “" & PUP 2 & 78 & 345 & 70 & 0.5 & 2 & " \\
\hline Draconids & GIA 1 & 196.3 & 171.8 & 30.7 & 0.717 & 3.51 & photographic \\
\hline " & GIA 2 & 196.0 & 177.0 & 25.0 & 0.700 & 3.33 & . \\
\hline “ & GIA 3 & 195.5 & 176.9 & 24.6 & 0.700 & 3.33 & “ \\
\hline Leonids & LEO 1 & 234.5 & 173.1 & 162.3 & 0.931 & 15 & “ \\
\hline “" & LEO 2 & 234.8 & 172.4 & 162.5 & 0.935 & 15 & " \\
\hline 55P/Tempel-Tuttle (1965 epoch) & $55 \mathrm{P} 1$ & 234.4 & 172.6 & 162.7 & 0.904 & 10.3 & \\
\hline 55P/Tempel-Tuttle (1998 epoch) & $55 \mathrm{P} 2$ & 234.6 & 172.5 & 162.5 & 0.905 & 10.3 & \\
\hline
\end{tabular}

Cygnids, on September 15, 1967. Of these, the kappa-Cygnids were the closest at $0.37 \mathrm{AU}$. We also note that Mariner 4 was within $0.17 \mathrm{AU}$ of the Giacobinid stream on October 30, 1967 (no spacecraft anomaly was reported at that time, however). It is difficult to believe that Mariner 4 could have actually encountered many stream meteoroids at the time in question, and in particular there is no good geometrical evidence, in the sense of a stream crossing, to support the idea of an encounter on 15 September, 1967. It is possible, but purely a point of conjecture, that Mariner 4 encountered a meteoroid stream that does not intersect the Earth's orbit.

During the December 10, 1967 anomaly, we need only consider one meteoroid stream-the Leonids. No other mean stream orbit is within $0.5 \mathrm{AU}$ of Mariner 4 at the time of the observed anomaly. The encounter conditions are shown in Fig. 5 (stream orbit characteristics are given in Table 12). Our new result is in contradiction to that of Haynes [55], who suggested that the anomaly related to the passage of Mariner 4 through the Southern Taurid stream. We find that Mariner 4 was some $0.84 \mathrm{AU}$ from the mean Southern Taurid stream orbit at the time of the anomaly. We also note that Haynes used what is now an old and inaccurate orbit for the stream (that given by Lovell[56]). At the time of the reported Mariner 4 anomaly the spacecraft was $0.07 \mathrm{AU}$ from the mean Leonid stream orbit. At this separation there is every reason to believe that the spacecraft may well have encountered some Leonid meteoroids. Indeed, an analysis by Levinson [57] has indicated that the roll error recorded by Mariner 4 can be explained on the basis of a few low-mass Leonid meteoroid hits.

The post-encounter orbit of Mariner 4 periodically placed it close to the mean orbit of the Leonid stream. During the 1966 Leonid storm, Mariner 4 was 2.2 AU away from the stream and in 1965 it was 1.6 AU from the stream. At these distance one would not expect any Leonid meteor- oid hits. Mariner 4 did pass within $0.1 \mathrm{AU}$ to the Leonid stream in March of 1966, however. Unfortunately, the telemetry recorded is incomplete from March 1966 to early 1967[53], and consequently a transient roll error anomaly may well have escaped detection. We note at this point that Mariner 4 exhausted its attitude-control gases on December 5, 1967 and it is possible, therefore, that the observed roll anomaly may have resulted from a number of different causes (e.g., solar radiation pressure). It is indeed unfortunate for our purposes that there is no supportative data on possible meteoroid hits at the time of the March 1966 closest approach between Mariner 4 and the Leonid stream.

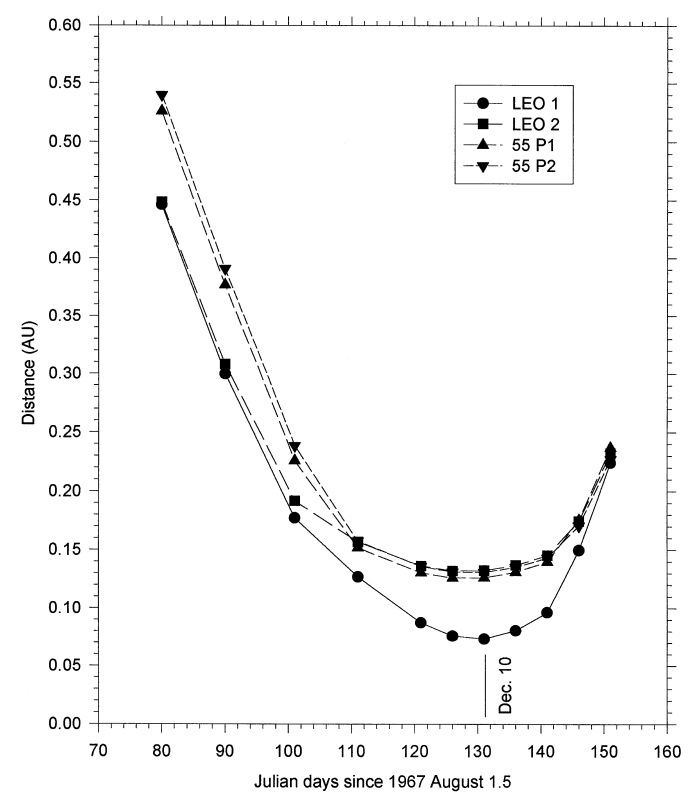

Fig. 5. Same as Fig. 3, but showing the encounter conditions for the Leonid meteoroid stream. 


\section{DISCUSSION}

Since the beginning of the space age there have been two meteor storms, and both of these storms were due to the Leonid stream. The Leonid storm of 1966 resulted in a meteoroid fluence of order $10^{-4}$ meteoroids $/ \mathrm{m}^{2}$ with masses greater than $10^{-5} \mathrm{~g}$. This constitutes the strongest meteor storm ever recorded. We have found no evidence to indicate that spacecraft operations were adversely affected during either the 1965 or the 1966 Leonid storms. Likewise, no on-orbit fragmentation events can be associated with these events. These results, however, are a direct consequence of the small surface areas presented by the majority of spacecraft on-orbit in the storm years. There is some compelling, but not conclusive evidence to indicate that the Pegasus II and III satellites recorded a Leonid meteoroid strike in 1965, and that the Mariner 4 spacecraft encountered low mass Leonid meteoroids in 1967.

Extrapolating our findings from the 1965-1966 epoch to the next Leonid storm epoch (that of 1998-2000) is not straightforward. The meteoroid fluences that might be encountered by spacecraft during the potential Leonid storms in 1998 and 1999 are not predictable at the present time. From an historical perspective (e.g., from Table 2) we might expect a ZHR of two to five thousand at storm maximum and a storm duration of about $5 \mathrm{~h}$. Under these circumstances a fluence $\sim 10^{-5}$ meteoroids $/ \mathrm{m}^{2}$, at a limiting mass of $10^{-5} \mathrm{~g}$, may well be realized. With this fluence, an impact probability of $\sim 0.01 \%$ will result for spacecraft with an exposed cross-sectional area of $10 \mathrm{~m}^{2}$. The present on-orbit US Space Command Satellite Catalogue contains about 8000 objects of which about $6 \%$ are functional satellites. With a fluence $\sim 10^{-5}$ meteoroids/ $\mathrm{m}^{2}$ we might expect, therefore, five to ten functional satellites to be hit by Leonid meteoroids (assuming an average spacecraft cross-section area of $20 \mathrm{~m}^{2}$, a mass index $s=2$, and a limiting meteoroid mass of $\left.10^{-7} \mathrm{~g}\right)$ during a storm. The consequences of such strikes are difficult to assess, but we note that a $10^{-7} \mathrm{~g}$ Leonid meteoroid will probably carry sufficient energy to puncture a typical solar array panel. Plasma and electrostatic discharge phenomena may also accompany Leonid meteoroid impacts.

Larger space platforms run a greater risk of being struck by meteoroids during a storm. The Mir space station, for example, has a surface area of $\sim 500 \mathrm{~m}^{2}$, and consequently an impact probability of order $50 \%$ could be realized during a Leonid storm (to a limiting meteoroid mass of $10^{-7} \mathrm{~g}$, and assuming a mass index of $s=2$ ). Since the Mir station has a greater level of impact shielding than most objects in LEO and GEO, it may not, in spite of its greater size, experience any significant meteoroid damage - its solar arrays, on the other hand, may well fare badly during a storm.
As a final comment, we note that the scheduling of human space flight missions, in which even very low impact probabilities are a concern, have been restricted by NASA during the times of potential Leonid storms $[55,56]$. This is a direct protection option that is unfortunately not available to the majority of spacecraft and it would seem only prudent, therefore, that all space platform operators develop a set of meteor storm contingency plans, not just for the 1999 Leonids, but for all future meteor storms.

Acknowledgements-This research was supported through grants from; The Institute for Space and Terrestrial Science, The Department of National Defence, the Defence Research Organization Ottawa, the Canadian Space Agency and TMI Communications. The assistance of Julie Cooper at the JPL archives is also gratefully acknowledged.

\section{REFERENCES}

1. Brown, P. and Jones, J., Earth, Moon, and Planets, 1995, 68, 223-245.

2. Cour-Palais, B. G., in Meteoroid environment model. NASA SP-8013, 1993.

3. Grun, E., Zook, H.A., Fechtig, H. and Giese, R.H., Icarus, 1985, 62, 244-272.

4. Divine, N.J., Journal of Geophysical Research, 1993, 98, 17029-17048.

5. Beech, M. and Brown, P., Monthly Notices of the Royal astronomical Society, 1993, 262, L35-L36.

6. Beech, M., Brown, P. and Jones, J., Quarterly Journal of the Royal Astronomical Society, 1995, 36, 127-152.

7. Koschack, R. and Rendtel, J., WGN, the Journal of the IMO, 1990, 18(4), 119-126.

8. Yeomans, D.K., Icarus, 1981, 47, 492-499.

9. Kresak, L., in Solid Particles in the Solar System, ed. I. Haliday and B. A. McIntosh. Reidel, Dordrecht, 1980, pp. 211-222.

10. Kazimiracak-Polonskaja, E. I., Astapovic, I. S. and Terenteva, A. K. 1968. The Leonid meteor stream. In Physics and Dynamics of Meteors. Kresak L, Millman P (Eds.) IAU Symposium 33. pp. 449-475.

11. McIntosh, B.A. and Millman, P., Meteoritics, 1970, 5, $1-18$.

12. Bronsten, V. A. 1968. The 1966 Leonids. In Physics and Dynamics of Meteors. Kresak L, Millman P (Eds.) IAU Symposium 33. pp. 440-445.

13. Hindley, K.B.J., Journal of the British Astronomical Association, 1970, 80, 223.

14. Jenniskens, P., Astronomy and Astrophysics, 1995, 295, 206-235.

15. Beech, M., Jones, J., Brown, P. and Webster, A.R., Advances in Space Research, 1997, 20(8), 1509-1512.

16. Brown, P. and Rendtel, J., Icarus, 1996, 124, 414-428.

17. Jacchia, J.G., Verniani, F. and Briggs, R.E., Smithsonian Contributions to Astrophysics, 1967, 17, 1139.

18. Millman, P., Journal of the Royal Astronomical society of Canada, 1970, 64, 55-57.

19. Porubcan, V. and Stohl, J., in Asteroids, Comets, Meteors 1991, ed. A. W. Harris and E. Bowell. Lunar and Planetary Institute, Huston, 1992, pp. 469-472.

20. Watson, F., Science, 1946, 104, 210.

21. Whipple, F., Astronomical Journal, 1947, 131, 131.

22. Hawkins, G., Annual Reiviews of Astronomy and Astrophysics, 1964, 2, 149-164. 
23. Nazarova, T. N. 1968. Meteoroid fluxes near Earth's orbit. In Physics and Dynamics of Meteors. Kresak L, Millman P (Eds.) IAU Symposium 33. pp. 382-387.

24. Zook, H., in Astronomy and Astrophysics Encyclopedia, ed. S. Maran. Van Nostrand, New York, 1992, pp. $441-444$.

25. Dycus, R.D. and Bradford, D.C., Observatory, 1965, 85, 88-89.

26. Dycus, R.D., Observatory, 1969, 89, 60-62.

27. McCrosky, R.E. and Posen, A., Smithsonian Contributions to Astrophysics, 1961, 4(2), 15-84.

28. Rendtel, J., Arlt, R. and McBeath, A., in IMO Monograph 2. Potsdam, 1995.

29. Nilsson, C., Science, 1966, 153, 1242.

30. Kellog, W.W., Planetary and Space Science, 1959, 1, 71-72.

31. Beech, M. and Toulmin, P. C., in Proceedings of the 4th International Conference on Tethers in Space, Vol. Vol. II. Smithsonian Institution, Washington, D.C., 1995, pp. 1233-1241.

32. Alexander, W.M., McCraken, C.W. and LaGow, H.E.J., Journal of Geophysical Research, 1961, 66, 3970-3973.

33. Brown, P., Jones, J. and Beech, M., in Proceedings of the 5th International Conference on Space '96, ed. S. W. Johnson. Published by the American Association of Civil Engineers, New York, 1996, pp. 13-19.

34. Berg, O.E. and Meredith, L.H.J., Journal of Geophysical Research, 1959, 66, 3970-3973.

35. Ferry, G.V., Blanchard, M.B. and Farlow, N.H.J., Journal of Geophysical Research, 1970, 75, 859-870.

36. Erickson, J.E.J., Journal of Geophysical Research, 1963, 74(2), 576-585.

37. Caswell, R.D., McBride, N. and Taylor, A., Journal of Impact Engineering, 1995, 17, 139.

38. McDonnell, T., McBride, N., Green, S.F., Zarnecki, J.C. and Taylor, A., Study report to the European Space Agency and British Aerospace, 1993.

39. Rendtel, J., WGN the Journal of IMO, 1993, 21(5), 235-239.
40. Hughes, D. W., in Cosmic Dust, ed. J. A. M. McDonnell. Wiley and Sons, Chichester, 1978, pp. 123-185.

41. Jehn, R., IAA 93-742, 1993, , 1-14.

42. Goldberg, L., The Astronomical Journal, 1961, 66 , 105-106.

43. Sky Telescope. 1963. 27. 183

44. Morrow, W.E. and MacLellan, D.C., The Astronomical Journal, 1961, 66, 107-113.

45. Johnson, N. and McKnight, D. S., in Artificial Space Debris. Orbit Book Company, Malabar, Florida, 1987.

46. Sky Telescope. 1966. 31. 350.

47. Naumann, R. J., in Pegasus satellite measurements of meteoroid penetration. NASA TM X-1192, 1966.

48. Clifton, S. and Naumann, R., in Pegasus satellite measurements of meteoroid penetration. NASA TM X1316, 1966.

49. Fechtig, H., Grun, E. and Kissel, J., in Cosmic Dust, ed. J. A. M. McDonnell. Wiley, Chichester, 1978, pp. 647-651

50. Anderson, H.R., Science, 1965, 149, 1226-1228.

51. 1966. Mariner Mars 1964 project report: Mission operations. NASA Technical Report \#32-881.

52. Alexander, W.M., McCraken, C.W. and Bohn, A., Science, 1965, 149, 1240-1241.

53. 1968. Mariner-Venus 1967: Final Report. NASA Report SP-190.

54. A. N. Other, Aviation Week and Space Technology, 1968, 22.

55. Haynes, N. R., in Determination of the meteor stream encountered by Mariner IV. JPL Inter office memo 312.3-26, 1968.

56. Lovell, A. C. B., in Meteor Astronomy. The Clarendon Press, Oxford, 1954.

57. Levinson, D.A.J., The Journal of Astronautical Sciences, 1977, 25, 129-142.

58. Loftus, J. P., NASA memo SL-95-186, 1995.

59. Levin, B. 1996. personal communication. 